\title{
LX. CORRESPONDENCE.
}

In Mind XXXII. Mr. J. Venn reviewn an article of mine on "A New Algebra of Logic" in Studies in Logic by Members of the Johns Hopkins University. In the course of this review he gives my solution of a certain gimple problem, and then proceeds to give what he calls "the solution as it might be worked out on Boole's plan". He gets as a conclusion, "All $d$ is either $a$ or $b$ or $c$ ". This he says is in reality the same as mine, which, relieved of a slight redundancy, is "There is some $b$ or $a$, or else all $d$ is $c^{\infty}$. Now, every reader must have seen that these two propositions are far from being the same. It seems to me worth while therofore to point out where the error lies, especially since it is a fundamental one, and moreover vitiates Mr. Venn's treatment of hypothetical propositions in his Symbolic Logic.

Mr. Venn says that he considers $\gamma \bar{\alpha}(1-a \beta)=0$ c to exactly express oymbolically" the hypothetical proposition "If no $a$ is $\beta$ all $\gamma$ is $\delta^{\prime}$; "for it expresses the fact that when $a \beta=0$ (vis., No $a$ is $\beta$ ) then $\gamma^{\bar{\delta}}=0$ (via., All $\gamma$ is $\delta)^{n}$. Of course, no one questions the fact that $\gamma(1-a \beta)-0$ (which Boole would have read 'All $\gamma$ which is not $\delta$ is both $a$ and $\beta$ ') implies the hypothetical proposition which Mr. Venn considers to be its "exact" meaning; but it implies 8189 other hypothetical propositions of the same kind. For Mr. Venn's principle of interpretation, generally expresed, seems to be as follows: If $X=P Q$ and $P$ and $Q$ contain no logical factor in common, then $X=0$ means 'If $\bar{Q}=0$ then $P=0$ '. Implies it of conrse, but it implies as well 'If $\bar{P}=0$ then $Q=0$,' and also a similar pair of hypothetical propositions for every other way of separating $X$ into two logical factors prime to each other. Thus $\gamma^{\bar{d}}$ $(1-a \beta)$ contains four class-symbols and has, when developed, three aggregant terms. It has therefore $Q^{4}=3$ logical prime factora, i.e, $\gamma \partial(1-a \beta)=(a+\bar{\beta}+\bar{\gamma}+\bar{\partial})(a+\bar{\beta}+\gamma+\bar{\delta})(a+\bar{\beta}+\gamma+\delta) . .$. There are thus $2^{12}-1$ ways of separating $\gamma \hat{\partial}(1-a \beta)$ into two logical factors prime to each other, and hence $2^{13}-8$, or 8190 , bypothetical propasitions which may be inferred from $\gamma \delta(1-a \beta)=0$; but even all these do not express its full logical content.

The problem above mentioned is as follows :- " What may be inferred independent of $x$ and $y$ from the two premisses 'Either some $a$ that is $x$ is not $y$, or all $d$ is both $x$ and $y$ ', and ' Either some $y$ is both $b$ and $x$, or all $x$ is either not $y$ or $c$ and not $b$ ' q These two digjunctive propositions are of course equivalent to the two hypothetical propositions ' If all $a$ that is $x$ is $y$, then all $d$ is both $x$ and $y$,' and 'If no $y$ is both $b$ and $x$, then all $x$ is either not $y$ or $c$ and not $b^{\prime}$. 'Mr. Venn expresses these as followr :-

$$
\begin{aligned}
& d(1-x y)(1-a x y)=0, \\
& x y(1-c b)(1-b x y)=0,
\end{aligned}
$$

and then reduces them to the simpler forms,

$$
\begin{gathered}
d(x \bar{y} \bar{a}+\bar{x})=0, \\
x y \bar{b}=0 .
\end{gathered}
$$

Eliminating $x$ and $y$ he gets the conclusion,

$$
d \bar{a} \bar{b}=0 \text {. }
$$

This conclusion he reads, as Boole would have read it, "All $d$ is either $a$ or 
$b$ or c'. But this is by no meang the valid conclusion from the premisses of the problem.

Mr. Venn's error lies in the fact that his symbolic statement of the first premiss may stand equally well for 2045 other hypothetical propositions, looking at it from his own point of view. Likewise his statement of the second premiss may stand equally well for 38,765 other hypothetical propositions. Lastly, his symbolic conclusion $d a b e=0$ may stand for 32,766 different hypothetical propositions when interpreted according to bis peculiar method. One of these hypotheticals is the proper conclusion from the premisses of the problem, since his symbolic premisses imply the premisses. It would have been remarkable if Mr. Venn had been able to tell which of the 32,768 is the correct concluaion. It is still more remarkable that he did not read dab $\bar{c}=.0$ as a hypothetical at all, but gives the Boolian interpretation of it. My conclusion, whose accuracy he does not impeach, shows how he might have read $d \bar{a} \bar{b} \bar{c}=0$ so as to get the proper conclusion from the premisses of the problem, vis., by dividing dabc into the two logical factors $\bar{c} \bar{b}$ and $d \bar{c}$, and equating to zero the negative of $\vec{a} b$ as the antecedent and equating to zero $d \vec{c}=0$ as the consequent. Thas we have 'If $\bar{a} \bar{b}=0$ then $d \bar{c}=0$,' that is, 'If there is no $a$ nor $b$, then all $d$ is $c$,' or ' Either $a$ or $b$ exists, or all $d$ is $c$ '.

In Mr. Venn's reprint of my solution there is a typographical error. The term $d x$ ahould read $d \dot{c}$.

O. H. Mitchell.

On the main point in question, vix, the inadequacy of the expression $\gamma(1-\alpha \beta)$ to express the proposition 'If no $a$ is $\beta$ then $\gamma$ is $\delta$,' I must quite admit the effectiveness of Mr. Mitchell's remarks. But I shall be glad, with the Editor's permission, to explain more fully on another occasion in what sense I proposed it, and to what extent I atill think that it may serve such a parpose. I will only remark here that I never in any published work clajmed the expression as exactly expressing the customary signification of such hypotheticals, though I certainly thought that it might, in certain cases, express more of such sigmification than I now see that it could do.

On the fundamental principle which anderlies the interpretation of these hypotheticals, viz, the impossibility of expressing particular propositions on $a 1$ and 0 scheme, I judge that I am entirely at one with Mr. Mitchell. I have urged-as against Boole and Jevons-as strongly as I could that such propositions demand some third symbol, whether it stand in the place of copula or predicate, and cannot be represented with the ordinary notation for universale.

J. VENN.

[Miss Ladd (Mrs Fabian Franklin), author of another paper in the Studies in Logic, also writes, with reference to Mr. Venn's Critical Notice in MIND XXXII., that the second sentence of the quotation on p. 589, which he found obscure, should have had the words "of the product" inserted after the words "partial inclusion".-EDrror] 